\title{
Type 2 diabetes and cardiometabolic risk may be associated with increase in DNA methylation of FKBP5
}

\author{
Robin Ortiz ${ }^{1}$, Joshua J. Joseph², Richard Lee ${ }^{3}$, Gary S. Wand ${ }^{4}$ and Sherita Hill Golden ${ }^{*^{*}}$ (D)
}

\begin{abstract}
Background: Subclinical hypercortisolism and hypothalamic-pituitary-adrenal (HPA) axis dysfunction are associated with type 2 diabetes (T2DM), cardiovascular disease, and metabolic dysfunction. Intronic methylation of FKBP5 has been implicated as a potential indicator of chronic cortisol exposure. Our overall objective in this study was to determine the association of chronic cortisol exposure, measured via percent methylation of FKBP5 at intron 2, with percent glycosylated hemoglobin ( $\mathrm{HbA1c}$ ), low-density lipoprotein cholesterol (LDL-cholesterol), waist circumference (WC), and body mass index (BMI), in a clinic-based sample of 43 individuals with T2DM.

Results: Greater percent methylation of the FKBP5 intron 2 at one CpG-dinucleotide region was significantly associated with higher $\mathrm{HbA} 1 \mathrm{c}(\beta=0.535, p=0.003)$ and $\mathrm{LDL}$ cholesterol $(\beta=0.344, p=0.037)$ and a second $\mathrm{CpG}$-dinucleotide region was significantly associated with higher BMI and WC $(\beta=0.516, p=0.001 ; \beta=0.403, p=0.006$, respectively).

Conclusions: FKBP5 methylation may be a marker of higher metabolic risk in T2DM, possibly secondary to higher exposure to cortisol. Further work should aim to assess the longitudinal association of FKBP5 with cardiovascular disease and glycemic outcomes in T2DM as a first step in understanding potential preventive and treatment-related interventions targeting the HPA axis.
\end{abstract}

Keywords: FKBP5, Methylation, Epigenetics, Cortisol, Diabetes, Cardiovascular disease, Obesity, Body mass index (BMI), Waist circumference, Hemoglobin A1c

\section{Background}

Subclinical hypercortisolism and hypothalamic-pituitaryadrenal (HPA) axis dysfunction are associated with type 2 diabetes (T2DM), cardiovascular disease, and metabolic dysfunction [1-3]. While recent research has shown alterations of the cortisol diurnal profile in those with T2DM, further research in this area has been hindered by lack of a chronic total cortisol (glucocorticoid) exposure measure. Recently, epigenetic modification of HPA axis-targeted genes has been identified as a potential measure of chronic exposure to cortisol and HPA axis dysfunction $[4,5]$. Understanding the role of HPA dysfunction, assessed via epigenetic modifications, in metabolic disorders, T2DM, and cardiovascular disease,

\footnotetext{
* Correspondence: Sahill@jhmi.edu

${ }^{4}$ Division of Endocrinology, Diabetes and Metabolism, Department of Medicine, Johns Hopkins University School of Medicine, 1830 E. Monument Street Suite 333, Baltimore, MD 21287, USA

Full list of author information is available at the end of the article
}

may lead to a unifying pathophysiology, thus setting the stage for preventive and therapeutic interventions targeting the HPA axis. It has been proposed that identifying a target, such as a specific modulator or receptor, could allow for restoration of HPA axis function to a homeostatic physiological state thereby reversing subclinical hypercortisolism and its associated comorbidities including metabolic syndrome, T2DM, and cardiovascular disease [6].

The FK506-binding protein $51 \mathrm{kDa}(F K B P 5)$ gene is a modulator, or co-chaperone, of the glucocorticoid receptor by limiting translocation of the receptor complex to the nucleus [7]. An observation that changes in FKBP5 methylation at intron 2 , specifically, were associated with shaping the human brain and function was one of the first demonstrations of the epigenetic interaction between genetics and environment [8]. FKBP5 has been linked to metabolic dysfunction including regulation of body weight after bariatric surgery and insulin resistance

(c) The Author(s). 2018 Open Access This article is distributed under the terms of the Creative Commons Attribution 4.0 International License (http://creativecommons.org/licenses/by/4.0/), which permits unrestricted use, distribution, and 
in non-diabetic individuals $[9,10]$. Epigenetic modification via loss of intronic methylation of FKBP5 has been associated with Cushing's syndrome and can serve as an indicator of chronic exposure to cortisol [11]. Cushing's can often manifest with cardiovascular and metabolic dysfunction including truncal obesity, hypertension, and glucose intolerance. FKBP5 methylation has also been associated with neuropsychiatric disease $[8,12]$. However, FKBP5 methylation has not been studied in individuals with T2DM, or in the context of specific measures of metabolic and cardiovascular disease. Given its importance in the function of the HPA axis via regulation of the glucocorticoid receptor, if FKBP5 is shown to be associated with metabolic syndrome, T2DM, and cardiovascular disease, it may serve as a promising target for interventions aimed at restoring HPA axis function.

Our overall objective in this study was to determine the association of chronic cortisol exposure, measured via percent methylation of $F K B P 5$ at, specifically, intron 2, as was studied by Klengel, et al. [8], with markers of metabolic and cardiovascular disease in those with T2DM. Specifically, the association of percent methylation of FKBP5 with percent glycosylated hemoglobin (HbA1c), low-density lipoprotein cholesterol (LDL cholesterol), body mass index (BMI), and waist circumference (WC), which is a surrogate for central obesity and an independent risk factor for cardiovascular disease [13]. We further explored the association between methylation of FKBP5 and (1) cardiovascular disease risk modifier, exercise, and (2) the presence of cardiovascular disease, assessed by the need for coronary artery disease intervention.

\section{Methods}

\section{Aims}

The aim of this study was to primarily assess methylation of FKBP5 at intron 2 with markers of metabolic and cardiovascular disease in those with T2DM including HbA1c, LDL cholesterol, BMI, and WC. A secondary aim was to assess the potential relationship between FKBP5 and exercise as well as a history of cardiovascular procedures in the study cohort.

\section{Study participants}

MinD, a study assessing the crude prevalence of minor depressive disorder (MinD) in a clinic-based population of 128 consented adults with T2DM, participant data was used for this analysis. Because the primary aim of the MinD study focused on data assessing minor depression prevalence and comorbidity with associated cardiovascular and metabolic measures in subjects with T2DM, control subjects without diabetes were not included in the original design. Further, participants taking antipsychotic medication or glucocorticoids were excluded as both medications may be associated with major mood disorders. The detailed methods of study design were previously described elsewhere and are described here briefly [14]. Individuals with physician-confirmed T2DM who were 18 years of age or older were recruited between 2011 and 2013 from the Diabetes Center clinics at Johns Hopkins Hospital during routine visits. Patients who were willing to participate in the study provided written informed consent. This study was approved by the Johns Hopkins University School of Medicine Institutional Review Board.

\section{Exposures and outcomes samples}

Baseline information was obtained during clinic visits using standardized questionnaires including demographics, race, level of education, and annual income. Calibrated devices were used by certified technicians and nurses to measure participants' weight, WC (average of two measurements around the umbilicus), and height. BMI was calculated as weight (kilograms)/height ${ }^{2}\left(\mathrm{~m}^{2}\right)$. Resting seated blood pressure (BP) was measured twice at 5-min intervals using an appropriately sized cuff with standard Hawksley random-zero instruments, and measurements were averaged for analysis. Hypertension was defined as systolic BP of $140 \mathrm{mmHg}$ or greater, diastolic BP of $90 \mathrm{mmHg}$ or greater, or use of antihypertensive therapy. HbA1c and lipid panels (including low-density lipoprotein, triglycerides, and high-density lipoprotein) were also collected and analyzed by Johns Hopkins Clinical Core Laboratory with LDL cholesterol calculated from total cholesterol.

A secondary aim of the MinD data collection was to assess the relationship of FKBP5 with measures of cardiometabolic risk in diabetes. Therefore, a subset of patients consented to provide blood samples to be used for future research purposes. Only subjects who did not consent to blood collection of DNA for research purposes were excluded. DNA was extracted from the blood and stored at $-20{ }^{\circ} \mathrm{C}$, until epigenetic analyses were conducted.

\section{Bisulfite pyrosequencing for DNA methylation (DNAm) quantification}

Two sets of bisulfite polymerase chain reaction (PCR) primers were designed to target each of two regions in the second intron of the human FKBP5 gene. The two regions flanked a glucocorticoid response element (GRE) [15] and contained consecutive cytosine-guanine dinucleotides (CpGs) implicated in previous studies $[8,11]$. The coordinates of the nine CpGs on chromosome 6 are Chr6: 35,606,441-35,606,662 (CpG1-4) and Chr6: 35,609,54235,609,666 (CpG5-9), according to the UCSC Genome Browser build GRCh37.hg19 assembly [16]. Two rounds of a nested PCR amplification were performed. In the first PCR, 3.5 $\mu \mathrm{L}$ bisulfate converted DNA was used. In the second PCR, $2 \mu \mathrm{L}$ template from the PCR was used. One of the nested bisulfite primers was biotinylated and HPLC-purified, allowing it to bind to sepharose beads and 
become single-stranded, in preparation for bisulfite pyrosequencing. The single-stranded amplicons were annealed to pyrosequencing primers and subjected to primer extension and nucleotide incorporation using the PyroMark Q96 MD pyrosequencer (Qiagen). The pyrosequencer QCPG program determined percent DNA methylation (DNAm) at all of the CPG dinucleotides downstream of the annealed primer at $>90 \%$ precision.

\section{Statistical analysis}

Subject demographics were analyzed using descriptive statistics. Outliers in methylation assays were identified if the value was greater than three standard deviations away from the mean suggesting sub- or supra-physiologic levels and likely erroneous. Multivariable linear regression was performed to examine the association of percent DNAm of FKBP5 (dependent variable) with HbA1c, LDL cholesterol, BMI, and WC (independent variables). Age, sex, and race were included as covariates in all regression analyses, based on previous cortisol analyses $[1,17]$. Beta regression coefficients with a $p$ value $<0.05$ were considered statistically significant. Given this was established as a hypothesis-generating study, no adjustments were made for multiple comparisons as such tests were considered too conservative for correlated hypotheses as we propose here. For all independent variables with significant beta regression coefficients, basic correlation $\mathrm{R}$ values were used to assess effect size using Cohen's effect size such that $r=0.1$ was considered of small effect size, $r=0.3$ was considered of medium effect size, and $r=0.5$ was considered of large effect size [18]. Based on preliminary results, a secondary analysis was completed including a multivariate regression with the number of cardiovascular procedures as a dependent variable and another with the number of days of physical activity greater than $30 \mathrm{~min}$ as an independent variable. All statistical analyses were performed using IBM SPSS Statistics Version 23.

\section{Results}

A total of 65 subjects had given consent for blood samples for epigenetic analysis. After excluding individuals with missing data on HbA1c $(n=5)$, LDL cholesterol $(n=11)$, BMI $(n=1)$, and WC $(n=11), 43$ participants were included in the analysis (Additional file 1: Figure S1). The participants were $48.8 \%$ female, $60.5 \%$ non-Hispanic black with a mean age of 61.9 years ( $\mathrm{SD} \pm 9.0$ years); other demographics are described in Table 1. Given that all participants had diabetes and were on anti-hypertensive medication, $81.4 \%$ of subjects with $\mathrm{WC} \geq 94 \mathrm{~cm}$ in men or $\geq 80 \mathrm{~cm}$ in women, therefore, met the National Cholesterol Education Program Adult Treatment Panel III (NCEP:ATPIII) criteria for metabolic syndrome [19].

We analyzed by pyrosequencing nine CpG-dinucleotides in intron 2 of $F K B P 5$ that were implicated in metabolic
Table 1 Participant demographic characteristics $(n=43)$

\begin{tabular}{ll}
\hline Characteristic & $\begin{array}{l}\text { Percentage or mean } \\
\text { (standard deviation) }\end{array}$ \\
\hline Sex (\%) & \\
$\quad$ Female & $48.8 \%(n=21)$ \\
Race (\%) & \\
$\quad$ Non-Hispanic White & $37.2 \%(n=16)$ \\
Non-Hispanic Black & $60.5 \%(n=26)$ \\
$\quad$ Asian & $2.3 \%(n=1)$ \\
Age (years) & $61.9 \pm 9.0$ \\
WC (cm) & $109.7 \pm 21.6$ \\
WC $\geq 94$ cm (men) or 80 cm (women) (\%) & $81.5 \%(n=35)$ \\
BMI (kg/m $\left.{ }^{2}\right)$ & $34.1 \pm 8.3$ \\
Antihypertensive medication use (\%) & $100 \%(n=43)$ \\
HbA1c (\%) & $8.3 \pm 1.6$ \\
LDL cholesterol (mg/dL) & $89 \pm 40$ \\
Cardiovascular procedure (\%)* & \\
Catheterization & $11.6 \%(n=5)$ \\
Percutaneous coronary intervention (PCl) & $4.7 \%(n=2)$ \\
Coronary artery bypass surgery (CABG) & $7.0 \%(n=3)$ \\
PCl and CABG & $4.7 \%(n=2)$ \\
\hline
\end{tabular}

${ }^{*}$ Collectively this represents the total number of individuals included in the FKBP5 analysis model with a history of cardiovascular procedures $(n=12)$

disorders in previous studies and flanked an experimentally validated GRE $[8,11]$. We observed a significant association between intronic FKBP5 DNAm in subjects with T2DM and multiple cardiometabolic outcomes in two different CpG-dinucleotides in intron 2 (Fig. 1). Greater percent DNAm of the FKBP5 intron 2 CpG9-dinucleotide (CpG9) (coordinates chr6: $35,609,542)$ was significantly associated with higher HbA1c $(\beta=0.535, p=0.003$, Fig. 1a) and LDL cholesterol $(\beta=0.344, p=0.037$, Fig. 1b). Greater percent DNAm of the FKBP5 intron 2 CpG7-dinucleotide (CpG7) (coordinates chr6: $35,609,628)$ was significantly associated with higher BMI and WC $(\beta=0.516, p=0.001$, Fig. $1 \mathrm{c}$, and $\beta=0.403, p=0.006$, Fig. $1 d$, respectively). The associations with $\mathrm{HbA1c}$, BMI, and WC were all of medium to large effect sizes (Table 2). These findings remained stable regardless of using the complete case sample $(n=43)$ or all available cases despite missing data (Additional file 2: Table S1, Additional file 3: Figure S2). Results for non-significant associations are shown in Additional file 2: Table S2.

In a secondary analysis, it was observed that having a history of more invasive cardiovascular procedures was associated with greater percent DNAm at CpG9 when adjusting for LDL cholesterol $(\beta=0.344, p=0.033)$ (Table 3). Greater percent DNAm at CpG7 was inversely associated with more days a week completing more than $30 \mathrm{~min}$ of physical activity even when controlling for WC $(\beta=-0.390, p=0.011)$ (Table 3). 

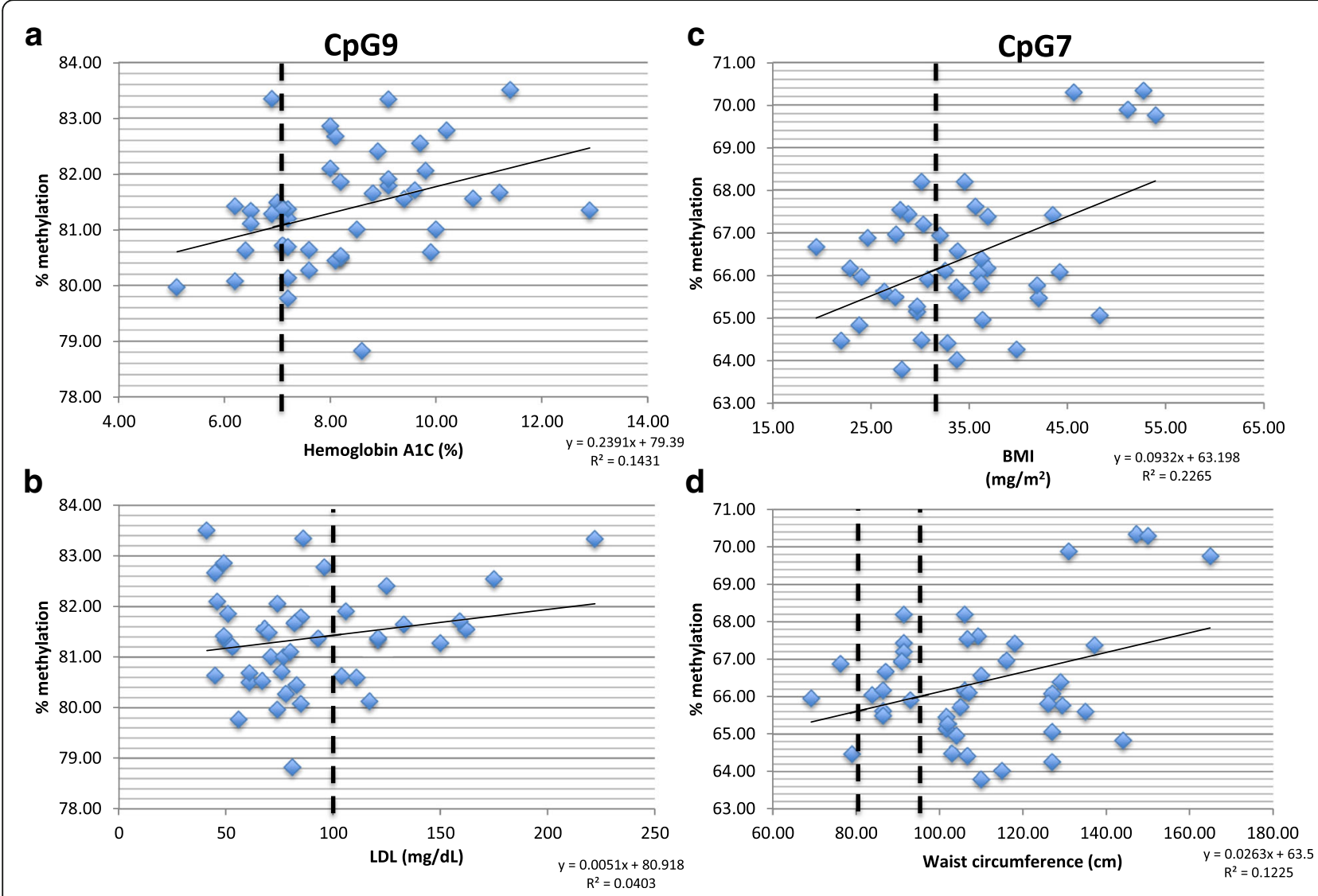

Fig. 1 FKBP5 methylation associated with cardiometabolic risk in individuals with diabetes. a Greater percent DNA methylation of the FKBP5 intron 2 CpG9-dinucleotide (CpG9) (coordinates chr6: 35,609,542) was significantly associated with higher HbA1c $(\beta=0.535, p=0.003)$, with a medium to large effect size $\left(R^{2}=0.143, R=0.378\right)$. Dotted line on the $x$-axis indicates target HbA1c of $7.0 \%$. $\mathbf{b}$ Greater percent DNA methylation of CpG9 was also significantly associated with higher $\operatorname{LDL}(\beta=0.344, p=0.037)$ with small to medium effect size $\left(R^{2}=0.040, R=0.201\right)$. Dotted line on the $x$-axis indicates target LDL of $100 \mathrm{mg} / \mathrm{dL}$. c Greater percent methylation of the FKBP5 intron 2 CpG7-dinucleotide (CpG7) (coordinates chr6: 35,609,628) was significantly associated with higher BMI $(\beta=0.516, p=0.001)$ with medium to large effect size $\left(R^{2}=0.227, R=0.476\right)$. Dotted line on the $x$-axis indicates a BMl cut-off for obesity at $\geq 30 \mathrm{~kg} / \mathrm{m}^{2}$. d Greater percent DNA methylation of CpG7 was also significantly associated with higher WC $(\beta=0.403, p=0.006)$ with medium to large effect size $\left(R^{2}=0.123, R=0.350\right)$. Dotted lines on the $x$-axis indicate cut-offs for $W C$ in metabolic syndrome criteria of $\geq 94 \mathrm{~cm}$ in men or $\geq 80 \mathrm{~cm}$ in women

\section{Discussion}

In our study of individuals with T2DM, we observed that one pyrosequenced $\mathrm{CpG}$-dinucleotide in intron 2 of the FKBP5 gene (CpG9) was significantly associated with higher HbA1c and LDL, and another CpG-dinucleotide
(CpG7) site with BMI and WC, though results should be interpreted in the context of a small sample size of 43 subjects. In the study population, mean HbA1c was above $7 \%$ (8.3\%), mean BMI was categorized as obese $\left(>30 \mathrm{~kg} / \mathrm{m}^{2}\right)$, there was a presence of hyperlipidemia (LDL cholesterol

Table 2 FKBP5 methylation associated with cardiometabolic risk in individuals with diabetes

\begin{tabular}{|c|c|c|c|c|}
\hline \multirow[t]{2}{*}{ Independent variables } & \multicolumn{2}{|c|}{ Model including the covariates age, sex, and race } & \multicolumn{2}{|c|}{ Effect size } \\
\hline & $\beta$ & $p$ & $R$ & Effect size \\
\hline \multicolumn{5}{|c|}{ Percent methylation of CpG9 } \\
\hline $\mathrm{HbA1c}$ & 0.535 & $0.003^{* *}$ & 0.378 & Medium-large \\
\hline LDL & 0.344 & $0.037^{*}$ & 0.201 & Small to medium \\
\hline \multicolumn{5}{|c|}{ Percent methylation of $\mathrm{CpG7}$} \\
\hline BMl & 0.516 & $0.001^{* *}$ & 0.476 & Large \\
\hline WC & 0.403 & $0.006^{* *}$ & 0.350 & Medium-large \\
\hline
\end{tabular}

The analysis included 43 subjects. Percent methylation of $\mathrm{CpG9}$ and $\mathrm{CpG} 7$ were the dependent variables in the model ${ }^{*} p<0.05 ;{ }^{* *} p<0.01$ 
Table 3 FKBP5 methylation associated with cardiovascular procedures and days of physical activity in individuals with diabetes

\begin{tabular}{llll}
\hline Dependent variable & Independent variable & $\begin{array}{l}\text { Model including the covariates age, sex, } \\
\text { and race }\end{array}$ & Model including additional covariate $^{\mathrm{a}}$ \\
\hline $\begin{array}{l}\text { Number of cardiovascular } \\
\text { procedures }\end{array}$ & Percent methylation of CpG9 & $\beta=0.247, p=0.112$ & LDL; $\beta=0.344, p=0.033^{*}$ \\
Percent methylation of CpG7 & Number of days of physical activity & $\beta=-0.492, p=0.001^{* *}$ & WC; $\beta=-0.390, p=0.011^{*}$ \\
\hline
\end{tabular}

${ }^{a}$ This model includes covariates of age, sex, and race in addition to the covariate listed below ${ }^{*} p<0.05 ;{ }^{* *} p<0.01$

greater than $100 \mathrm{mg} / \mathrm{dL})$ in $30 \%$ of subjects, and a majority of subjects (81.4\%) were above target WC and thus met criteria for metabolic syndrome. This suggests that the associations between DNAm of FKBP5 and the outcome measures for cardiovascular and metabolic dysfunction observed in our study population including HbA1c, LDL cholesterol, BMI, and WC, are clinically meaningful as risk for disease. The relationship between FKBP5 methylation, a surrogate marker for presumed chronic cortisol exposure, glycemic control in T2DM, and cardiovascular disease (CVD) risk, is notably indicated by the medium to large effect size of the association between intron 2\% DNAm with HbA1c and WC, and the medium effect size of the relationship with LDL cholesterol. Our secondary analysis further suggests that the association of
FKBP5 with CVD as methylation of FKBP5 is also associated with cardiovascular interventions including catheterization, percutaneous intervention (PCI), and coronary artery bypass grafting (CABG), and inversely associated with exercise.

There is a higher incidence of CVD in individuals with diabetes due to both macrovascular pathology and associated comorbid metabolic disorders, including hyperlipidemia [20, 21]. However, current treatments continue to target individual disease processes. Though our findings are correlative and cannot be used to determine causality, they support our hypothesis of a potential unifying pathophysiology of underlying hypercortisolism related to activation of glucocorticoid receptor signaling (Fig. 2), which may implicate the glucocorticoid pathway

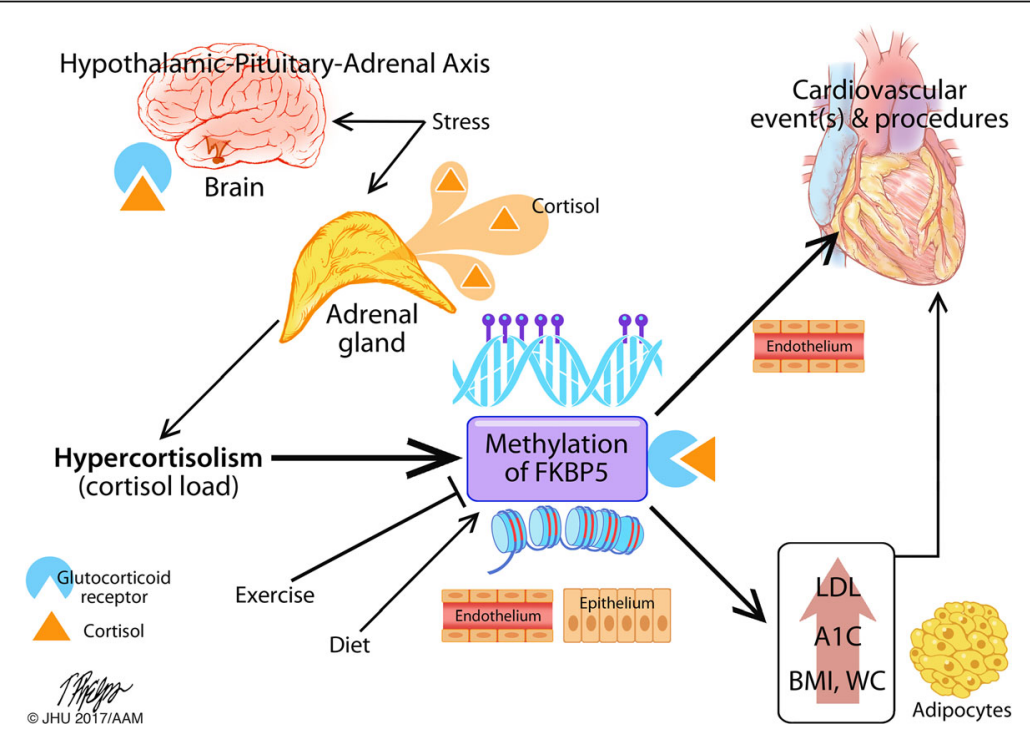

Fig. 2 The hypothesized relationship of FKBP5 DNA methylation and cardiometabolic risk. Our findings demonstrate evidence that FKBP5, and therefore likely the hypothalamic-pituitary-adrenal axis, play a role in metabolic and cardiovascular disease risk and outcome. FKBP5, and the glucocorticoid receptor, is expressed in the brain, intestinal endothelial, and epithelial tissues. FKBP5 expression in the brain (hypothalamus and hippocampus) as well as these other tissues has been associated previously with the influences of stress and cortisol load, exercise, and diet. Prior demonstration has also shown the function of FKBP5 to play a role in adipogenesis and in endothelial changes in myocardial infarction. Taken together, these associations are hypothesized to link to risk and outcomes in cardiometabolic disease through methylation of FKBP5, and therefore, potentially expression or sensitivity of the glucocorticoid receptor. The methylation of FKBP5 is associated with increased clinical risk factors for disease including hyperlipidemia (elevated LDL), chronic hyperglycemia (elevated HbA1c), and obesity (high BMI and WC) in individuals with diabetes. Further, methylation of FKBP5 may be associated with cardiovascular procedures and inversely associated with exercise independent of LDL and obesity. Though many clinical trials have established the classical model of pathophysiologic associations with diabetes and hyperglycemia, hyperlipidemia, obesity, and cardiometabolic disease, the findings illustrated here demonstrate that there may be a role for the hypothalamic-pituitary-adrenal axis and glucocorticoid pathway dysfunction in the underlying pathophysiology of cardiometabolic disease risk and outcomes 
as a new potential target for preventive and therapeutic interventions.

Our study corroborates current literature demonstrating the significance of FKBP5 in associations with metabolic dysfunction and dysglycemia. For example, a polymorphism, rs1360780, has been associated with insulin resistance in children and adolescents with obesity and also with lower weight loss up to 26 months after bariatric surgery in adults [10, 22]. Studies have also demonstrated that FKBP5 protein expression is responsive to certain environmental stimuli. For example, animal studies have shown that higher levels of FKBP5 expression in the hypothalamus are related to increases in body weight and, further, hypothalamic, hippocampal, and intestinal colon epithelial expression of $F K B P 5$ may be responsive to dietary intake and environmental stress [23-25]. In humans, adipose tissue $F K B P 5$ expression and insulin resistance increase with dexamethasone exposure [9].

The observations in our secondary analysis that higher FKBP5 methylation is inversely associated with current exercise activity and positively associated with a history of cardiovascular procedures are also supported by the literature. It has been demonstrated that FKBP5 may play a role in vascular endothelial function in exercise in obese individuals as well as on endothelial platelet aggregation in individuals with acute myocardial infarction [26-28]. This dysfunction may be the underpinnings of the associations between cortisol and coronary artery calcifications, mortality after cardiac surgery (CABG), and all-cause CVD mortality [29-31]. Similarly, elevated hair cortisol levels as a measure of chronic cortisol exposure predict myocardial infarction and heart failure severity [32, 33].

Epigenetics is the concept that the genome may be modifiable through environmental exposures. One method of epigenetic modification is methylation of CpG-dinucleotide sites of DNA. One study demonstrated that a decrease in methylation of an intron 2 site of FKBP5 is present in Cushing's syndrome [11]. Only one study to our knowledge, an animal study, exists assessing methylation and expression of FKBP5 as a result of chronic steroid exposure, which showed persistent loss of methylation correlating with increased steroid exposure [4]. These studies suggest hypomethylation in association with chronic glucocorticoid exposure and our study identified hypermethylation associated with risk factors for CVD in T2DM. However, we note that two of the FKBP5 intronic CpGs analyzed by Klengel et al. also showed increase in DNAm associated with the trauma exposure [8]. While gain of methylation events are usually associated with gene silencing, there are notable examples, such as at imprinted loci, where increase in DNAm inhibit binding of insulator proteins such as CTCF that govern complex enhancer-promoter interactions and gene activation [34]. If lower FKBP5 expression is the outcome of hypermethylation in this group, it could indicate that these subjects are more responsive at the tissue level to cortisol since lower FKBP5 protein expression allows more cortisol signaling. The increased responsiveness in adipose and other tissues could be the reason for the correlation $[9,24,25,28]$. In this scenario, methylation is not a marker of cortisol exposure but of cortisol sensitivity. Additional in vitro experiments are needed to identify methylation-dependent binding of disease-specific factors whose presence in intron 2 may increase expression of FKBP5 and its relationship with chronicity of cortisol exposure. Though further studies are needed to elucidate these gene loci-specific environment interactions, taken together with the findings in the current literature, our study may suggest that chronic exposure to glucocorticoids in obesity and T2DM may be in part responsible for associated cardiovascular and metabolic dysfunction through FKBP5 expression.

Our study has many strengths. First, our sample includes a clinically generalizable sample of patients with T2DM and clinical outcome measures relevant to the assessment of disease in this population, especially $\mathrm{HbA1c}$ as a marker of glucose control, LDL cholesterol as a marker of increased risk of CVD, and BMI and WC as markers of a diagnosis of metabolic syndrome. Second, our study is one of the first studies to explore the epigenetics of FKBP5 in humans with T2DM, in relation to metabolic and CVD risk and clinical characteristics including exercise and cardiac procedures. Finally, there is strength in the specificity of selecting a region of interest within the FKBP5 gene, intron 2. This offers a specific target for future studies and potentially future interventions.

Our study does have some potential limitations. First, this is a small sample size and only includes individuals with T2DM seen in a tertiary care practice without a control group, which limits generalizability. The small sample size also limits the ability to adjust for multiple confounders and permits risk of overfitting the data in analyses. Given the small sample size and that the FKBP5 analysis was hypothesis generating as a secondary aim of the MinD study, it may have been underpowered to accurately assess the effect sizes listed in Table 2 . Also, control subjects without T2DM were not included in the original design. Future studies should include larger samples with control subjects in order to more stringently establish, and then validate, methylation as a potential biomarker for chronic glucocorticoid exposure. Second, our study is correlative and does not offer insight into causative association between glucocorticoid receptor function and disease outcomes. Third, we do not have longitudinal cortisol measures to assess the association with percent methylation as a surrogate for chronic hyper- or hypocortisolism nor did we measure 
peripheral $F K B P 5$ protein expression and, therefore, cannot assess whether the increase in DNA methylation we observe is associated with FKBP5 or cortisol levels. Therefore, we propose that future studies of greater sample size, including a matched control group, assess both chronic, longitudinal assessment of cortisol load, such as hair cortisol levels, and RNA expression of FKBP5 protein. We also assessed peripheral blood samples for FKBP5 methylation and not adipocytes, epithelial, or endothelial cells. Finally, in our statistical analyses, our associations were interpreted without corrections for multiple comparisons, as typical multiple corrections assume that the tests are independent and are too conservative for correlated hypotheses as we have tested here.

\section{Conclusion}

In conclusion, we examined FKBP5 methylation among individuals with T2DM and found that FKBP5 DNAm was associated with higher levels of HbA1c, LDL cholesterol, BMI, and WC, which are all risk factors for CVD. We also found that increased percent FKBP5 methylation was associated with greater number of cardiac procedures, supporting our initial finding of the relationship between FKBP5 and adverse cardiovascular risk factors. Thus, FKBP5 DNAm may be a marker of higher cardiovascular risk in T2DM, possibly secondary to higher exposure to cortisol. There is a critical need for further characterization of the longitudinal association of FKBP5 with CVD in T2DM as a first step in understanding potential causation, and future directions for preventive and treatment interventions targeting the HPA axis.

\section{Additional files}

\section{Additional file 1: Figure S1. Consort diagram. (PPTX $18 \mathrm{~kb}$ ) \\ Additional file 2: Table S1. FKBP5 methylation associated with cardiometabolic risk does not differ by missing data case exclusion. Table S2. FKBP5 percent methylation at various CpG-dinucleotide sites in intron 2 is not associated with cardiometabolic risk. (DOCX $20 \mathrm{~kb}$ ) \\ Additional file 3: Figure S2. FKBP5 methylation associated with cardiometabolic risk in individuals with diabetes including all available data. (PPTX $28 \mathrm{~kb}$ )}

\section{Abbreviations}

BMI: Body mass index $\left(\mathrm{kg} / \mathrm{m}^{2}\right)$; BP: Blood pressure: CABG: Coronary artery bypass graft; Chr: Chromosome; CpG: Cytosine guanine dinucleotide; CVD: Cardiovascular disease; DNA: Deoxyribonucleic acid; DNAm: DNA methylation; FKBP5: FK506-binding protein 51 kDa gene; GRE: Glucocorticoid response element; HbA1c: Glycosylated hemoglobin (\%); HPA: Hypothalamicpituitary-adrenal; HPLC: High-performance liquid chromatography; LDL: Lowdensity lipoprotein cholesterol (mg/dL); MinD: Minor depressive disorder; PCR: Polymerase chain reaction; T2DM: Type 2 diabetes mellitus;

TG: Triglycerides; WC: Waist circumference $(\mathrm{cm})$

\section{Acknowledgements}

The authors would like to acknowledge Timothy Phelps, Professor and Medical Illustrator, Graduate Program of Art as Applied to Medicine, Johns Hopkins in Baltimore, Maryland, for his help in the design of Fig. 2 in our manuscript.

\section{Funding}

The project described was supported by Award Number Grant UL1TR001070 from the National Center For Advancing Translational Sciences. The content is solely the responsibility of the authors and does not necessarily represent the official views of the National Center For Advancing Translational Sciences or the National Institutes of Health.

This study was supported by the National Institute of Diabetes, Digestive, and Kidney Diseases (R03 DK088997 awarded to SHG) and the National Institute of Alcohol Abuse and Alcoholism (U01 AA020890 awarded to GSW).

\section{Availability of data and materials}

Individuals with physician-confirmed T2DM who were 18 years of age or older were recruited between 2011 and 2013 from the Diabetes Center clinics at Johns Hopkins Hospital during routine visits. The datasets used and/or analyzed during the current study are available from the corresponding author on reasonable request.

\section{Authors' contributions}

$\mathrm{RO}$ contributed to study design, data analysis, manuscript writing, and manuscript revising. JJ was involved in project design, data gathering, data analysis, and manuscript revising. RL was responsible for laboratory analyses, data analysis, and manuscript revising. GW contributed to the original study design, laboratory analyses, data analysis, and manuscript revising. SG obtained funding and contributed to the original study design, project design, data gathering, data analysis, and manuscript revising. All authors read and approved the final manuscript.

Ethics approval and consent to participate

Patients who were willing to participate provided written informed consent This study was approved by the Johns Hopkins University School of Medicine Institutional Review Board.

\section{Competing interests}

The authors declare that they have no competing interests.

\section{Publisher's Note}

Springer Nature remains neutral with regard to jurisdictional claims in published maps and institutional affiliations.

\section{Author details}

${ }^{1}$ Departments of Internal Medicine \& Pediatrics, Johns Hopkins University School of Medicine, 600 North Wolfe Street, Harvey Bldg. Rm 805, Baltimore, MD 21287, USA. ²Division of Endocrinology, Diabetes, and Metabolism, Department of Medicine, The Ohio State University Wexner Medical Center, 566 McCampbell Hall, 1581 Dodd Drive, Columbus, OH 43210, USA

${ }^{3}$ Department of Psychiatry and Behavioral Sciences, Johns Hopkins University School of Medicine, 720 Rutland Ave. Ross Bldg. 1068, Baltimore, MD 21205 , USA. ${ }^{4}$ Division of Endocrinology, Diabetes and Metabolism, Department of Medicine, Johns Hopkins University School of Medicine, 1830 E. Monument Street Suite 333, Baltimore, MD 21287, USA.

Received: 2 November 2017 Accepted: 5 June 2018 Published online: 19 June 2018

References

1. Joseph JJ, Wang X, Spanakis E, Seeman T, Wand G, Needham B, Golden SH. Diurnal salivary cortisol, glycemia and insulin resistance: the multi-ethnic study of atherosclerosis. Psychoneuroendocrinology. 2015;62:327-35.

2. Nederhof E, van Oort FV, Bouma EM, Laceulle OM, Oldehinkel AJ, Ormel J. Predicting mental disorders from hypothalamic-pituitary-adrenal axis functioning: a 3-year follow-up in the TRAILS study. Psychol Med. 2015; 45:2403-12

3. Joseph JJ, Golden SH. Cortisol dysregulation: the bidirectional link between stress, depression, and type 2 diabetes mellitus. Ann N Y Acad Sci. 2017; 1391:20-34

4. Lee RS, Tamashiro KL, Yang X, Purcell RH, Harvey A, Willour VL, Huo Y, Rongione M, Wand GS, Potash JB. Chronic corticosterone exposure increases expression and decreases deoxyribonucleic acid methylation of Fkbp5 in mice. Endocrinology. 2010;151:4332-43. 
5. Lee RS, Tamashiro KL, Yang X, Purcell RH, Huo Y, Rongione M, Potash JB, Wand GS. A measure of glucocorticoid load provided by DNA methylation of Fkbp5 in mice. Psychopharmacology. 2011;218:303-12.

6. Gragnoli C. Hypothesis of the neuroendocrine cortisol pathway gene role in the comorbidity of depression, type 2 diabetes, and metabolic syndrome. Appl Clin Genet. 2014;7:43-53.

7. Cattaneo A, Riva MA. Stress-induced mechanisms in mental illness: a role for glucocorticoid signalling. J Steroid Biochem Mol Biol. 2016;160:169-74.

8. Klengel T, Mehta D, Anacker C, Rex-Haffner M, Pruessner JC, Pariante CM, Pace TW, Mercer KB, Mayberg HS, Bradley B, et al. Allele-specific FKBP5 DNA demethylation mediates gene-childhood trauma interactions. Nat Neurosci. 2013;16:33-41.

9. Pereira MJ, Palming J, Svensson MK, Rizell M, Dalenback J, Hammar M, Fall T, Sidibeh CO, Svensson PA, Eriksson JW. FKBP5 expression in human adipose tissue increases following dexamethasone exposure and is associated with insulin resistance. Metabolism. 2014;63:1198-208.

10. Hartmann IB, Fries GR, Bucker J, Scotton E, von Diemen L, KauerSant'Anna M. The FKBP5 polymorphism rs1360780 is associated with lower weight loss after bariatric surgery: 26 months of follow-up. Surg Obes Relat Dis. 2016;12:1554-60.

11. Resmini E, Santos A, Aulinas A, Webb SM, Vives-Gilabert Y, Cox O, Wand G, Lee RS. Reduced DNA methylation of FKBP5 in Cushing's syndrome. Endocrine. 2016;54:768-77.

12. Argentieri MA, Nagarajan S, Seddighzadeh B, Baccarelli AA, Shields AE. Epigenetic pathways in human disease: the impact of DNA methylation on stress-related pathogenesis and current challenges in biomarker development. EBioMedicine. 2017;18:327-50.

13. Klein S, Allison DB, Heymsfield SB, Kelley DE, Leibel RL, Nonas C, Kahn R, Association for Weight Management and Obesity Prevention, NAASO, et al. Waist circumference and cardiometabolic risk: a consensus statement from shaping America's health: Association for Weight Management and Obesity Prevention; NAASO, the Obesity Society; the American Society for Nutrition; and the American Diabetes Association. Diabetes Care. 2007;30:1647-52.

14. Golden SH, Shah N, Naqibuddin M, Payne JL, Hill-Briggs F, Wand GS, Wang NY, Langan S, Lyketsos C. The prevalence and specificity of depression diagnosis in a clinic-based population of adults with type 2 diabetes mellitus. Psychosomatics. 2017:58:28-37.

15. Klengel T, Binder EB. FKBP5 allele-specific epigenetic modification in gene by environment interaction. Neuropsychopharmacology. 2015;40:244-6.

16. Aken BL, Achuthan P, Akanni W, Amode MR, Bernsdorff F, Bhai J, Billis K, Carvalho-Silva D, Cummins C, Clapham P, et al. Ensembl 2017. Nucleic Acids Res. 2017;45:D635-42.

17. Hajat A, Diez-Roux A, Franklin TG, Seeman T, Shrager S, Ranjit N, Castro C, Watson K, Sanchez B, Kirschbaum C. Socioeconomic and race/ethnic differences in daily salivary cortisol profiles: the multi-ethnic study of atherosclerosis. Psychoneuroendocrinology. 2010;35:932-43.

18. Cohen J. A power primer. Psychol Bull. 1992;112:155-9.

19. Kassi E, Pervanidou P, Kaltsas G, Chrousos G. Metabolic syndrome: definitions and controversies. BMC Med. 2011:9:48.

20. Tehrani DM, Zhao Y, Blaha MJ, Mora S, Mackey RH, Michos ED, Budoff MJ, Cromwell W, Otvos JD, Rosenblit PD, Wong ND. Discordance of low-density lipoprotein and high-density lipoprotein cholesterol particle versus cholesterol concentration for the prediction of cardiovascular disease in patients with metabolic syndrome and diabetes mellitus (from the multiethnic study of atherosclerosis [MESA]). Am J Cardiol. 2016;117:1921-7.

21. Katz R, Budoff MJ, O'Brien KD, Wong ND, Nasir K. The metabolic syndrome and diabetes mellitus as predictors of thoracic aortic calcification as detected by non-contrast computed tomography in the multi-ethnic study of atherosclerosis. Diabet Med. 2016;33:912-9.

22. Fichna M, Krzysko-Pieczka I, Zurawek M, Skowronska B, JanuszkiewiczLewandowska D, Fichna P. FKBP5 polymorphism is associated with insulin resistance in children and adolescents with obesity. Obes Res Clin Pract. 2016;12(1):62-70.

23. Balsevich G, Uribe A, Wagner KV, Hartmann J, Santarelli S, Labermaier C, Schmidt MV. Interplay between diet-induced obesity and chronic stress in mice: potential role of FKBP51. J Endocrinol. 2014:222:15-26.

24. Zhang L, Qiu B, Wang T, Wang J, Liu M, Xu Y, Wang C, Deng R, Williams K, Yang Z, et al. Loss of FKBP5 impedes adipocyte differentiation under both normoxia and hypoxic stress. Biochem Biophys Res Commun. 2017:485:761-7.
25. Chen Q, Swist E, Beckstead J, Green J, Matias F, Roberts J, Qiao C, Raju J, Brooks SP, Scoggan KA. Dietary fructooligosaccharides and wheat bran elicit specific and dose-dependent gene expression profiles in the proximal colon epithelia of healthy Fischer 344 rats. J Nutr. 2011;141:790-7.

26. Laughlin MH, Padilla J, Jenkins NT, Thorne PK, Martin JS, Rector RS, Akter S, Davis JW. Exercise training causes differential changes in gene expression in diaphragm arteries and 2A arterioles of obese rats. J Appl Physiol (1985). 2015;119:604-16.

27. Eicher JD, Wakabayashi Y, Vitseva O, Esa N, Yang Y, Zhu J, Freedman JE, McManus DD, Johnson AD. Characterization of the platelet transcriptome by RNA sequencing in patients with acute myocardial infarction. Platelets. 2016;27:230-9.

28. Zhao J, An Q, Goldberg J, Quyyumi AA, Vaccarino V. Promoter methylation of glucocorticoid receptor gene is associated with subclinical atherosclerosis: a monozygotic twin study. Atherosclerosis. 2015;242:71-6.

29. Kumari M, Shipley M, Stafford M, Kivimaki M. Association of diurnal patterns in salivary cortisol with all-cause and cardiovascular mortality: findings from the Whitehall II study. J Clin Endocrinol Metab. 2011;96:1478-85.

30. Ronaldson A, Kidd T, Poole L, Leigh E, Jahangiri M, Steptoe A. Diurnal cortisol rhythm is associated with adverse cardiac events and mortality in coronary artery bypass patients. J Clin Endocrinol Metab. 2015;100:3676-82.

31. Vogelzangs N, Beekman AT, Milaneschi Y, Bandinelli S, Ferrucci L, Penninx BW. Urinary cortisol and six-year risk of all-cause and cardiovascular mortality. J Clin Endocrinol Metab. 2010;95:4959-64.

32. Pereg D, Chan J, Russell E, Berlin T, Mosseri M, Seabrook JA, Koren G, Van Uum S. Cortisol and testosterone in hair as biological markers of systolic heart failure. Psychoneuroendocrinology. 2013;38:2875-82.

33. Pereg D, Gow R, Mosseri M, Lishner M, Rieder M, Van Uum S, Koren G. Hair cortisol and the risk for acute myocardial infarction in adult men. Stress. 2011;14:73-81.

34. Bell AC, Felsenfeld G. Methylation of a CTCF-dependent boundary controls imprinted expression of the lgf2 gene. Nature. 2000;405:482-5.

Ready to submit your research? Choose BMC and benefit from

- fast, convenient online submission

- thorough peer review by experienced researchers in your field

- rapid publication on acceptance

- support for research data, including large and complex data types

- gold Open Access which fosters wider collaboration and increased citations

- maximum visibility for your research: over $100 \mathrm{M}$ website views per year

At BMC, research is always in progress.

Learn more biomedcentral.com/submissions 